\title{
An Efficient Compound Image Compression Using Optimal Discrete Wavelet Transform and Run Length Encoding Techniques
}

DOI 10.1515/jisys-2016-0096

Received July 1, 2016; previously published online July 24, 2017.

\begin{abstract}
Reduction in file size leads to reduction in the number of bits required to store it. When data is compressed, it must be decompressed into its original form bit for bit. Compound images are defined as images that contain a combination of text, natural (photo) images and graphic images. Here, compression is the process of reducing the amount of data required to represent information. Image compression is done on the basis of various loss and lossless compression algorithms. This research work deals with the preprocessing and transformations used to compress a compound image to produce a high compression ratio (CR), less compression time and so on. In the compression process the images are considered for preprocessing and discrete wavelet transform with adaptive particle swarm optimization process. The purpose of this optimization technique is to optimize the wavelet coefficient in Harr wavelet for improving the CR value. In the image compression process, run length coding is used to compress the compound images. Based on this technique, it produces minimum $\mathrm{CR}$ and less computation time of compound images.
\end{abstract}

Keywords: Image compression, run length encoding, optimization, digital compound images, transformation.

\section{Introduction}

Image compression is the use of data compression on digital images. As a result, the goal is to decrease excess of the image data keeping in mind the end goal to have the capacity to store or transmit data in an effective form [17]. Image compression systems assume a vital part in numerous applications with constrained assets for review, storage and processing [14]. On preparing transfer speed, number of operations expands every second henceforth urging the scope and compression of colored images. Different application with particular color spaces have been introduced, though a compression method evident to the human visual framework is exceptionally anticipated [20]. Compression methods can be arranged into lossy and lossless compression techniques. The advantage of lossy over lossless is to decrease encoding and decoding time, compression ratio (CR) and energy in case of power compelled applications [7]. In lossy compression, the first signal cannot be precisely reproduced from the compressed data. The reason is that a significant part of the subtle element in an image can be disposed of without incredibly changing the presence of the image [6]. Despite that, the lossless plan is reversible, and this represents an image signed with the littlest conceivable number of bits without loss of any data and the CR accomplished is low, for example, LOCO-I, CALIC, JPEG-LS and JPEG2000 [25]. Two image compression algorithms investigating this thought are examined. The first depends on the benchmark discrete cosine transform (DCT)-based JPEG, while the second uses the discrete wavelet transform (DWT)-based JPEG. The standard JPEG and DWT-based variant of JPEG are still broadly utilized for compression of still images accessible on the Web and created by digital cameras

*Corresponding author: Priya Vasanth Sundara Rajan, Research Scholar, Department of Computer Science, Bharathiyar University, Coimbatore, India, e-mail: priyavasanths0289@gmail.com

A. Lenin Fred: Mar Ephraem College of Engineering and Technology, Mallankara Hills, Elavuvilai, Marthandam, India 
[9]. Lossy image compression goes for diminishing the document size of an image while corrupting the image quality as meager as would be prudent. A standout amongst the most noticeable and broadly utilized lossy image compression algorithms today is JPEG, which depends on the DCT [15]. Due to the constraints of capacity and transmission transfer speed of the images, the primary issue of the innovation lies in how to pack a colossal measure of visual information into a low piece rate stream, on the grounds that the measure of medical image data would overpower the storage device without an effective compression scheme [8]. While these plans concentrate on encrypting the images, they do not have any compression capacity. Here, a chaos-based joint image compression and encryption algorithm utilizing DCT and SHA-1 are proposed [23]. The wavelet compression technique connected to images may be regularly helpful in telemedicine to decrease the bandwidth required for transmitting illustrations records for examination or remote diagnostics. Images are framed by pixels that are little pictures whose color is related to a numerical worth as indicated by the order used [3]. This compression technique goes for getting high $\mathrm{CR}$ without influencing the quality of an image. Wavelet compression uses thresholding strategy with a specific end goal to remove certain wavelet coefficients in this compressing of the image [18]. Efficient compression and improved transmission procedures for taking care of medical images are of most extreme significance. For the compression of volumetric medical datasets it is demonstrated that 3D wavelet-based codes beat the DCT-based arrangements while giving required functionalities, for example, quality and determination adaptability, random access and ROI coding [4].

A bit-field preserving coding consolidated with run length encoding (RLE) to pack the wavelet coefficients was proposed. The proposed technique has the benefit of straightforward and forward information processing [5]. The image ought to be effectively compressed lossless in the transmission and capacity. Normally utilized lossless compression techniques incorporate Huffman coding, LZW coding, arithmetic coding, bit-plane coding, etc [12]. The LUCAS ${ }^{\mathrm{TM}} 2$ is a completely pneumatic automatic device that is outfitted with a drag for compression and a suction cup for dynamic decompression and should be appropriately set in the central breast region [2]. Combining quad-tree system, neighbor look and asymptotic procedure is necessary to execute a quick coding strategy. Contrasted with Furao's no search technique, the CR, the quality and encoding time are all enhanced greatly [22]. Keeping in mind that the end goal is to decrease the computational assets required by a quantum computer, we utilize NASSRP and an algorithm to actualize lossless quantum compression. The simulation results demonstrated that NASSRP encourages the lossless quantum compression of binary images [13].

\section{Literature Review}

In 2014, Wang et al. [21] had anticipated the productive and secure prerequisites of image transmission in various exploration works done to encrypt the compressed image. A plan is proposed to encrypt the compressed image by chaotic map and arithmetic coding. It upgrades the security of arithmetic coding as well as enhances the CR. With a specific end goal to have high sensitivities of key and plain image, the keys that are utilized to decide the parameter and initial value of chaotic maps are identified with the plain image. The trial results approve the impact of the proposed plot and show that the compressed and encrypted image is secure and helpful for transmission.

In 2014, Zhou et al. [24] proposed the current approaches to encrypting images in light of compressive detecting, for the most part, regarding the entire measurement matrix as the key, which renders the key too expensive to convey and retain or store. To take care of this issue, a new image compression-encryption hybrid algorithm is proposed to acknowledge compression and encryption all the while, where the key is effortlessly conveyed, put away or remembered. The measurement matrices in compressive utilizing is to detect the circulate matrices and controlling the first column vectors of the circulant frameworks with a logistic guide. Simulation results confirm the adequacy, security of the proposed algorithm and the worthy compression performance. 
In 2013, Agulhari et al. [1] suggested a compression method, taking into account the decision of a wavelet that minimizes the contortion of compression for every electrocardiogram considered. The scaling filter utilized for the determination of the wavelet capacity is obtained from the determination of an optimization problem, which is unconstrained since the scaling filter is parameterized in a manner that the imperatives connected to the scaling filter are implanted on the parameters. The bitmap that educates the positions of the held coefficients is encoded alongside the estimations of the coefficients by utilizing an enhanced version of the RLE system.

In 2013, Saadi et al. [16] proposed a mixed software/hardware implementation of 2-D signals encoder/ decoder utilizing dyadic DWT in light of quadrature mirror filters utilizing quick wavelet Mallat's algorithm. This work is composed and aggregated on the embedded development kit EDK6.3i and the synthesis software ISE6.3i, which is accessible with Xilinx Virtex-IIV2MB1000 FPGA. Huffman coding plan is utilized to encode the wavelet coefficients with the goal that they can be transmitted logically through an Ethernet transmission control protocol/Internet protocol based association. The conceivable reconfiguration can be misused to achieve higher execution. The configuration will be incorporated into the neutron radiography framework that is utilized with the Es-Salem research reactor.

In 2013, Kaur et al. [10] suggested that image compression is a standout amongst the most noticeable utilizations of wavelets. We inspected and compared different wavelet-based procedures, for example, consistent wavelet transform, DWT, wavelet packets and fast wavelet transform. The DWT analyzes the signal at different frequency bands with distinctive resolutions by disintegrating the signal into an estimate and detail data. The image coded by DWT does not have the issue of blocking curios which the DCT methodology may endure. Mallet algorithm based fast wavelet investigation utilizes augmentation of a given limited length signal and evaluates the outskirt impacts because of convolution.

In 2012, Singh and Sharma [19] anticipated that image compression means decreasing the size of illustrations record, without trading off on its quality. Two procedures are lossy methods and lossless methods. Digital imaging and video assume an essential part in image processing along these lines; it is important to add to a framework that creates a high level of compression while preserving critical image/video. The DWT and DCT usage, on the grounds that these are the lossy procedures, furthermore present Huffman encoding method which is lossless. The outcomes demonstrate that the proposed hybrid algorithm performs vastly improved in terms of peak signal to noise ratio (PSNR) with a higher CR contrasted with stand-alone DCT and DWT algorithms.

\section{Proposed Methodology}

Image compression is the application of data compression on digital images. In effect, the objective is to reduce redundancy of the image data in order to store or transmit data in an efficient form. This image compression work considers the compound images as input to apply the adaptive median filter to preprocessing steps to remove the noise. After removal of unwanted noises, the process applies DWT to transform the spatial domain to frequency domain process. Most of the visually significant information about the image is concentrated in a few coefficients of the DWT process. Then the run length coding (RLC) process is applied to encode the image to find the $\mathrm{CR}$ in original and encoded image. Improving CR optimizes the wavelet coefficient value using a different optimization technique such as genetic algorithm (GA), particle swarm optimization (PSO) and adaptive particle swarm optimization (APSO). This method contributes groupings of identical symbols (pixels), as it keeps running by smaller symbols. RLC is utilized as a part of the images. RLC is an basic type of information compression in which running of information are put away as a single data value and count, instead of first run. After getting the minimum CR, the decoding process will be applied to reconstruct the image with the help of inverse DWT. The proposed plan accomplishes the maximum accuracy and the minimum computational compression time, and this procedure will be executed utilizing the MATLAB software. Figure 1 shows the proposed block diagram of image compression process. 


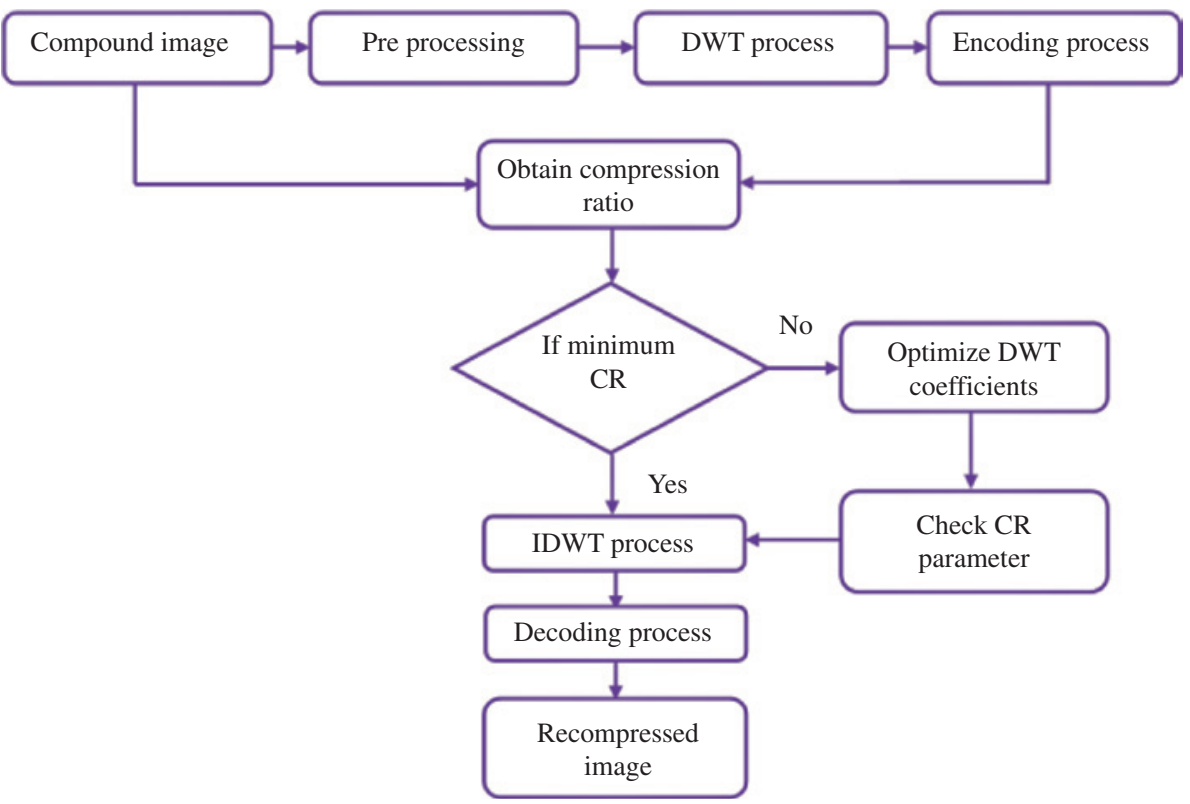

Figure 1: Block Diagram for Image Compression and Decompression Process.

\subsection{Compound Image}

Compression of a compound image is more critical than the natural image. Basically, a compound image is a combination of text, graphics and pictures. Compound images are a different kind of images that contain both palletized regions, which have text or graphics and continuous tone regions. Examples of compound images are shown in Figure 2.

\subsection{Preprocessing}

The adaptive median filter is used to empower the flexibility of the filter to change the size as needs to be founded on the guess of local noise density. The adaptive median filter depends on a trans-conductance comparator, in which immersion current can be altered to go about as a local weight operator. The purpose of filtering in this process is, if the image is noisy and target pixels neighboring pixel worth is somewhere around 0's and 255's then we supplant pixel esteem with the median value.

Let $S_{i, j}$, which locates at $(i, j)$, be the gray intensity of an $R \times C$ image $S$ and $\min \max L \times L$ be the dynamic range of $S$ i.e. mini, $j \max L \leq x \leq L$ for all $(i, j)$ which accords to the following rule:

$$
(i, j) \in A=\{1 \ldots \ldots . . R\} \times\{1 \ldots \ldots . C\}
$$

In the conventional noise model, we assume $y$ is the noisy image; the model is given by

$$
y_{i, j}= \begin{cases}L_{\min } & \text { with percentage } p \\ L_{\max } & \text { with percentage } q \\ S_{i, j} & \text { with percentage } 1-p-q\end{cases}
$$

where rate $=p+q$ means the noise level in the image, and assume that the filtering window $W_{i, j}$ is a window of size $(2 C+1) \times(2 C+1)$ centered at a position at $(i, j), W_{i, j}$ and can be written as 


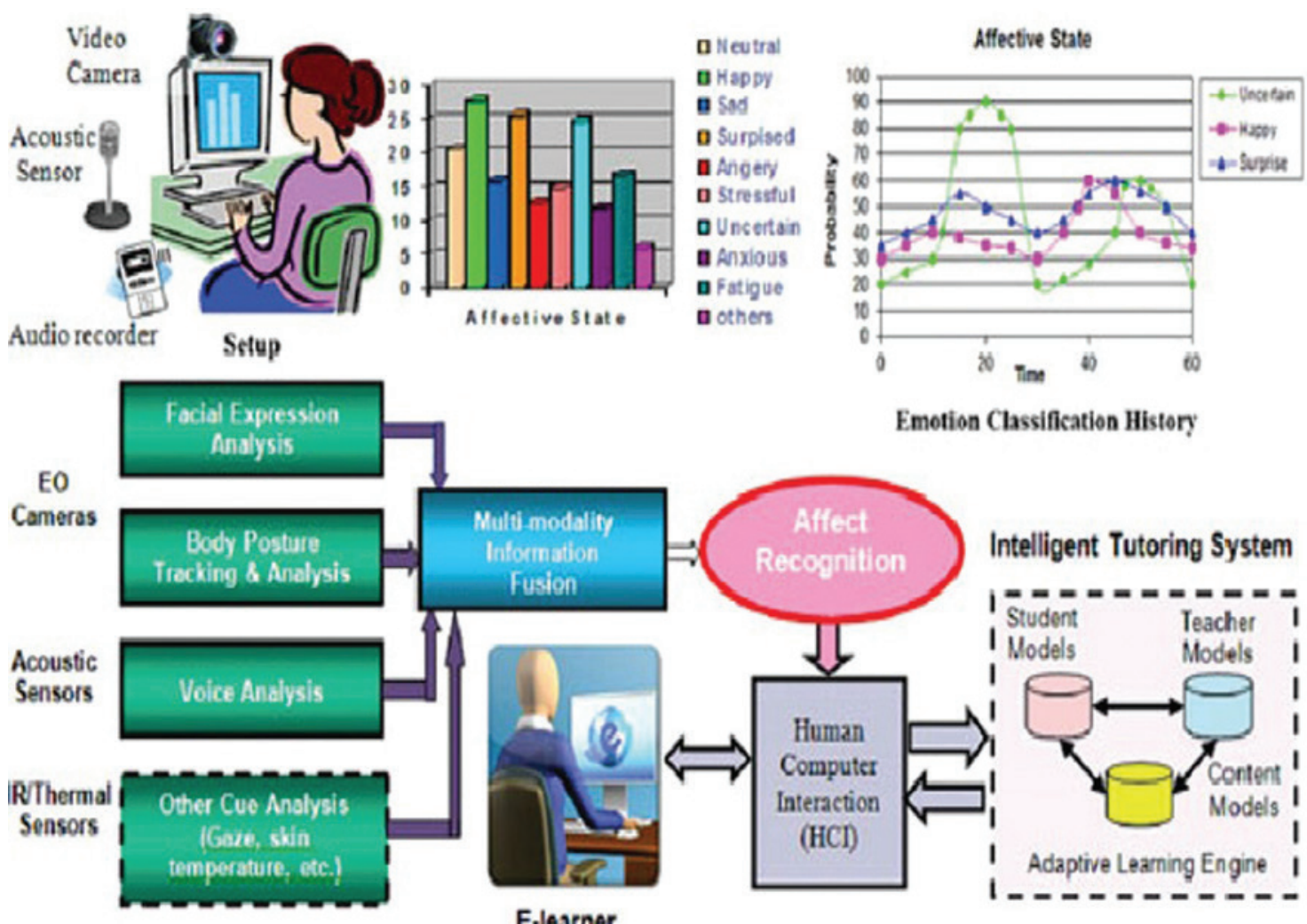

Figure 2: Example for Compound Image.

$$
W_{i, j}=\left\{s_{i-C, i-C} \ldots \ldots . . x_{i, j}, \ldots \ldots \ldots . x_{i+C, j+C}\right\}
$$

Let $w=2 C+1 \leq W \max$. The filter tries to improve the output image $y_{i, j} y$ the median in the window.

\subsection{Discrete Wavelet Transform}

It is computationally impossible to analyze a signal using all wavelet coefficients, so one may wonder if it is sufficient to pick a discrete subset of the upper half plane to be able to reconstruct a signal from the corresponding wavelet coefficients. The DWT consists of decomposition and reconstruction filters. Some most popular wavelet filters are Daubechies, Coiflets, Symlets and Harr wavelets. Using the DWT in compression process improves CR level of the compound image [11].

These functions are generated by the translation and dilation of a unique function, known as the "wavelet mother" denoted by $\delta(t)$ and is given by equation (1).

$$
\delta_{m, n}(t)=a^{-m / 2} \delta\left(a^{-m} t-n b\right)
$$

The corresponding discrete subset of the half plane consists of all the points $\left(a^{n}, n a^{m} b\right)$ with $m, n$ in $z$. A sufficient condition for the reconstruction of any signal $x$ of finite energy is shown by the formula

$$
x(t)=\sum_{m \in z} \sum_{n \in z}\left(x, \delta_{m, n}\right) . \delta_{m, n}(t)
$$

The wavelet transform has the advantage of achieving both spatial and frequency localizations. DWT is applied in the image and the image is converted to four bands. After one level of decomposition, there will be four frequency bands, in particular, the low-low (LL), low-high (LH), high-low (HL) and high-high (HH); the decomposition process is shown in Figure 3. 


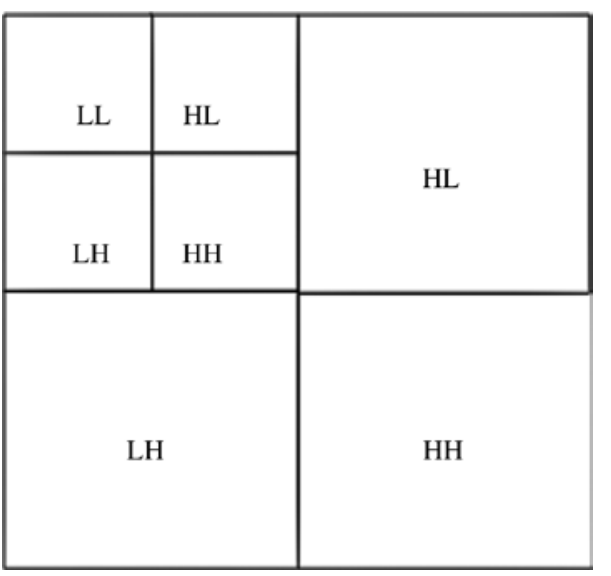

Figure 3: Image Decomposition.

\subsubsection{Wavelet Functions}

(a) Daubechies wavelets

$$
\varphi(t)=\sum_{n=0}^{N-1} a_{n} \varphi(2 x-n)
$$

where the sequence $\left(a_{0}, a_{1}, \ldots . . a_{N-1}\right)$ of real numbers is called a scaling sequence or scaling mask; the wavelet proper is obtained by a similar linear combination.

(b) Coiflet wavelet

Coiflet scaling functions also exhibit vanishing moments. In coifN, $\mathrm{N}$ is the number of vanishing moments for both the wavelet and scaling functions. These filters are also referred to in the literature by the number of filter taps, which is $2 \mathrm{~N}$.

(c) Symlet wavelet

Symlet wavelet (SYM), also known as "least asymmetric" wavelet, defines a family of orthogonal wavelets. SYM is defined for any positive integer $\mathrm{n}$. The scaling function $\varphi(t)$ and wavelet function $\delta(t)$ have compact support length of $2 n$. The scaling function has $n$ vanishing moments.

(d) Haar wavelet

In mathematics, the Haar wavelet is a sequence of rescaled "square-shaped" functions which together form a wavelet family or basis. The Haar wavelet's mother wavelet function $\delta(t)$ can be described as

$$
\delta(t)=\left\{\begin{array}{rl}
1 & 0 \leq t \leq 1 / 2 \\
-1 & 1 / 2 \leq t \leq 1 \\
0 & \text { otherwise }
\end{array}\right.
$$

Its scaling function $\varphi(t)$ can be described as

$$
\varphi(t)= \begin{cases}1 & 0 \leq t \leq 1 \\ 0 & \text { otherwise }\end{cases}
$$

This process minimum CR is attained in Harr wavelet coefficients in DWT process, so for better CR optimize Harr coefficients using optimization techniques. 


\subsection{Optimal Module for Compression Process}

In this module, the input compound images are transformed to the wavelet domain by the use of optimized DWT. The wavelet high-pass and low-pass filter Harr coefficients are optimized using the different optimization GA, PSO and APSO algorithm among this minimum CR obtained in APSO process.

\section{Pseudo code for APSO}

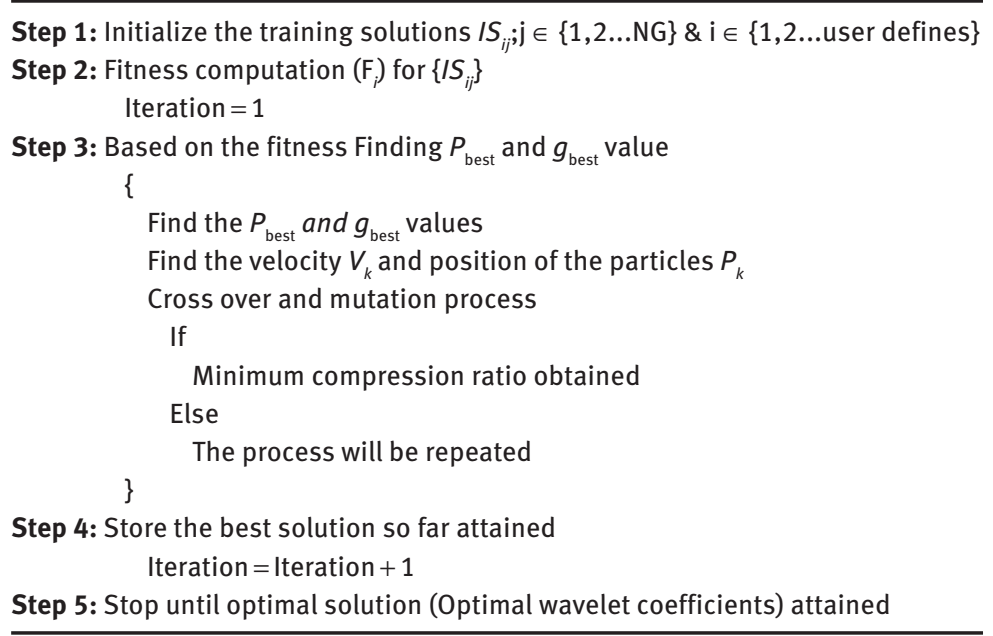

\subsubsection{Initialization Process}

The input parameters such as hidden layer and neuron is an initial solution and $\mathrm{i}$ is a number of solutions. This process is known as initialization process. Initialize the wavelet coefficients range from 0 to 1 .

\subsubsection{Fitness Computation}

The process of fitness computation is applied to equation (8) to find the fitness for the individual solution, and this process is computed as follows:

$$
\text { Compression ratio }=\left[\frac{\text { Compressed file size }}{\text { Actual file size }}\right] \star 100
$$

\subsubsection{New Population Update by Using Following Procedure}

Finding the $p_{\text {best }}$ and $g_{\text {best }}$ value

In the start, the fitness value is calculated approximately for every particle. The optimal one is considered as the $p_{\text {best }}$ and $g_{\text {best }}$ value among the fitness value. After that iteration, the current optimal fitness value chooses the $p_{\text {best }}$ and the overall best fitness value chooses the $g_{\text {best }}$.

Find the velocity $V_{k}$ and position of the particle $P_{k}$.

Now in PSO, equally the best velocity is initiated by all particles and the best positions are established by every particle in the search method.

$$
\begin{gathered}
V_{k}^{y+1}=w \cdot V_{k}^{y}+h_{1}{ }^{\star}{ }_{1}{ }^{\star}\left(\text { Pbest }_{k}-P_{k}^{y}\right)+h_{2}{ }^{\star} r_{2}{ }^{\star}\left(\text { Gbest }_{k}-P_{k}^{y}\right) \\
P_{k}^{y+1}=P_{k}^{y}+V_{k}^{y+1}
\end{gathered}
$$


where $V_{k}$ is the particle velocity, $P_{k}$ is the current particle, $h_{1}$ and $h_{2}$ are the learning factors, and $r_{1}$ and $r_{2}$ are the random value within the $[0,1]$.

In the wake of discerning the velocity and position estimation of the particles, the fitness value is again ascertained for the recently assessed velocity of the particles. In order to make the optimization more efficient, crossover and mutation operations are prepared once the velocity and position are assessed.

\section{Crossover}

Reproduction contains the crossover and mutation operation. The crossover operation has many methods to produce the offspring. They are one point, two points, uniform and arithmetic crossover in these process single point crossovers used. A crossover operates by randomly selecting a crossover point within a chromosome, then interchanging the two parent chromosomes between these points to produce two new offspring. During the recombination of chromosomes, it avoids genes at the head and tails are spited.

In parents 1 and 2 chromosomes, green and brown color are modified in their positions, and the outstanding genes of the chromosomes are interchanged between the parent chromosomes. This process is demonstrated in Figure 3.

\section{Mutation}

After that, the child chromosomes are altered for raising the effectiveness of the solution, and the bold letter illustrates the modified gene on the chromosome. Alteration is the process of creating new offspring from the single parent and preserves the variety of every chromosome illustrated in Figure 4. There is a possibility to find the gene of a child to modify arbitrarily. This gene process is better than the old parents. The mutation has two techniques such as random and alternate technique.

- Consecutively occurring zeros in the input data stream are very common.

- Non-zero digits with same value occurring consecutively is a very rare case in classic compound image compression.

The optimized RLE algorithm is used to identify the presence of a zero or consecutively occurring zeros in the input data stream. The presence of continuous zero digits in the input data stream toggles the initialized value of variable once. The variable is reinitialized at the end of the continuous zero digits in the input data stream. After getting the optimal wavelet coefficients, the encoding process will be applied here. The RLC is used for compression and decompression process.

\subsection{Run Length Encoding}

This is a very simple form of data compression in which runs of data (that is, sequences in which the same data value occurs in many consecutive data elements) are stored as a single value and count, rather than like

Parent 1

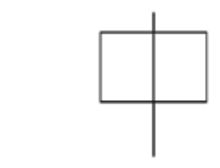

Parent 2

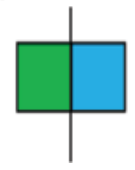

Offspring 1

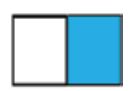

Offspring 2

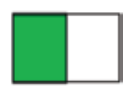

Figure 4: Single Point Crossover. 
Offspring 1

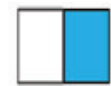

Offspring 2

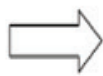

New offspring 2
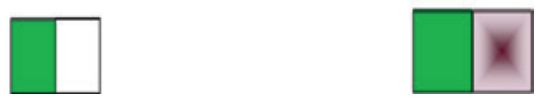

Figure 5: Mutations.

an original run. This is a very simple compression method used for sequential data. It is very useful for the data that contains simple graphic images like icons, line drawing and animations. For the single occurrence of a particular value, frequency was not used because that would cause an overhead affecting the compression efficiency. The consecutive information is exceptionally favorable on account of the repeating information as shown in Figure 5.

\section{Sample processing example}

- $\quad$ Input stream: 33333333252525858585

- $\quad$ Output stream: 433325385

The output stream produced a series of frequency-pixel value pairs as previously discussed. This new representation of original matrix information for individual matrices was forwarded to the next section for processing. Figure 6 shows the flow chart for RLC process in image compression.

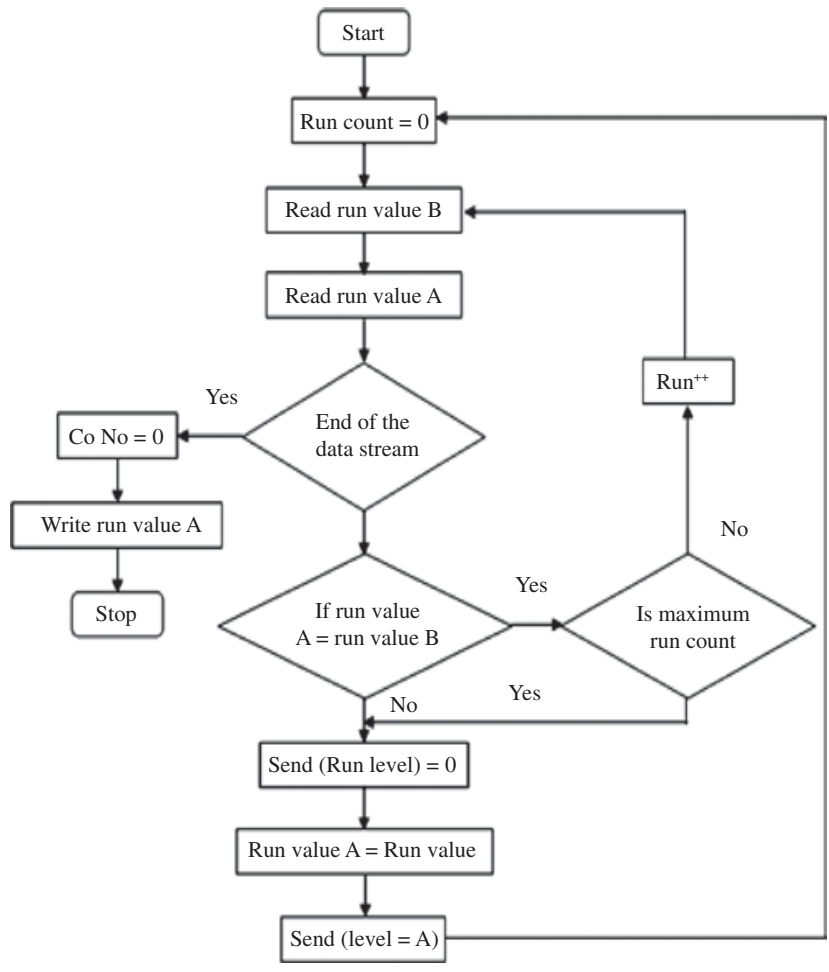

Figure 6: Flow Chart for RLC Process. 
The encoded message for a fixed coding scheme consists of an 8-bit value of RUN and an 8-bit signed value of LEVEL. This kind of scheme is called byte level run length coding.

\section{RLC compression and decompression procedure}

\section{Compression Algorithm}

Step 1: Start on the first element of input A

Step 2: Initialize the values with count $=l, k=0$

Step 3: Read the first element of input data $A$

Step 4: As the value of $K$ is 0 'it will print the input data $A$ and then it increments the $K$ value to 1 '

Step 5: Again it goes to step 3 takes the second data $B$ and next the checks the value of $k$

Step 6: As the value of $k$ is 1 it now it will checks for whether data $A=$ data $B$, if the data $A=$ data $B$ it will Increment the count value if not equal it prints the count value after the data 1 value and again goes to step 2

Decompression Algorithm

Step 1: Start on the first element of the data input

Step 2: Read the data A and initialize count with 1

Step 3: Print the data which is in register 1

Step 4: Take the second data from input B

Step 5: If Count $=B$ then go to step 2 else print $A$ and increment Count and repeat

\section{Result and Discussion}

In this section, the experimental setup, the evaluation metrics employed and the simulation results obtained for the proposed model are discussed. Here consider the different compound images to the image compression process using transformation and compression techniques. The technique is implemented in MATLAB on a system having $8 \mathrm{~GB}$ RAM and $3.2 \mathrm{GHz}$ Intel i-7 processor.

\subsection{Performance Measures}

\subsubsection{Compression Ratio}

The CR is defined as the ratio between the sizes of original image to size of the compressed image. This ratio gives how much compression is achieved for a particular image and usually indicates the picture quality. Generally, the higher CR results, the poorer the quality of the resulting image. The tradeoffs between CR and picture quality is an important one to consider the compressing images.

\subsubsection{Compression Size}

Compression size and original size are defined as the amount of time required to compress and decompress a picture, respectively.

\subsubsection{Mean Square Error}

Mean square error (MSE) measures the square error between the original and the compressed images.

$$
\text { Mean square error: } \mathrm{MSE}=\frac{1}{M N} \sum_{i=0}^{M-1} \sum_{j=0}^{N-1}\left[I(i, j)-I^{\prime}(i, j)\right]
$$

where $M \times N$ is the size of the image, and $I(i, j)$ and $I^{\prime}(i, j)$ are the matrix elements of the decompressed and the original image at $(i, j)$ pixel. 


\subsubsection{Peak Signal to Noise Ratio}

The PSNR is calculated between the input original image and the reconstructed image. PSNR is most commonly used to measure the quality of reconstruction of loss compression codec's (e.g. for image compression). The formula for PSNR is given as

$$
\text { PSNR }=10 \log _{10} \frac{p_{\text {max }}^{2}}{\text { MSE }}
$$

Here, max is the maximum possible pixel value of an image.

\subsection{Database Description}

The experimentation is done in three types of compound images, which are scanned document images, computer generated images and photographic images with compound nature. The images are collected from different documents and the web.

\subsubsection{Analysis Results in Compression Process}

This section shows the analysis result in compression process, and also we consider different images from the database. Based on this analysis, the compression process calculates the PSNR, MSE and CR.

Table 1 shows the original image, decompressed image and CR for different compound images. All the image files that we have tested are of different sizes. The CR of the proposed algorithm is better than that of our existing algorithms. Also, from the result, this algorithm is able to compress very large images that JPEG failed to compress compared to proposed technique.

Table 2 shows the original file size, compressed file size, MSE and PSNR of eight different images for proposed method DWT with Harr wavelet coefficients APSO optimization process. Maximum PSNR is 41.69 in image 3; the MSE of that image is 4.4035, and file compressed size difference is 1300 , similar difference in other images. So compression rate is increasing, and memory space is decreasing. This technique is more useful at the time when redundancy data is high. For example, redundancy data has been occuring in video and image compression.

Figures 7-9 show the comparison chart for parameters PSNR, MSE and CR. These graphs help to find the optimal value by comparing the optimization techniques (APSO, PSO and GA) and without optimization techniques in image compression process. The proposed APSO compression process has obtained a minimum value of 0.94 shown in Figure 9; compared to other techniques, the difference is $0.25 \%$ in PSO and $0.58 \%$ in GA. When image 2 compared with image 3 the APSO technique (0.922) with GA difference is $0.02 \%$ and also $0.23 \%$ optimization process. Figure 8 shows the PSNR comparison of proposed and other techniques; the maximum PSNR is 40.77 with similar values attained in other images. Hence, for computing the total difference in all algorithms the average value is $2.35 \%$. Then Figure 7 shows the MSE value comparison in all techniques; the minimum error value is 4.82 in APSO process.

Figure 10 illustrates one set of input values of the procedure during the performance technique that is carried out in the MATLAB programming indicated on the relative graph. This figure shows the input image and decompressed images with PSNR, MSE and CR in the different optimization algorithm. In this graphical user interface (GUI) based approach, the input values are changed, and the corresponding outputs are evaluated.

\section{Conclusion}

Image compression plays a significant role in reducing the transmission and storage cost. All the image compression techniques are useful in their related areas, and every day a new compression technique is 
Table 1: Comparison of Reconstructed Images.

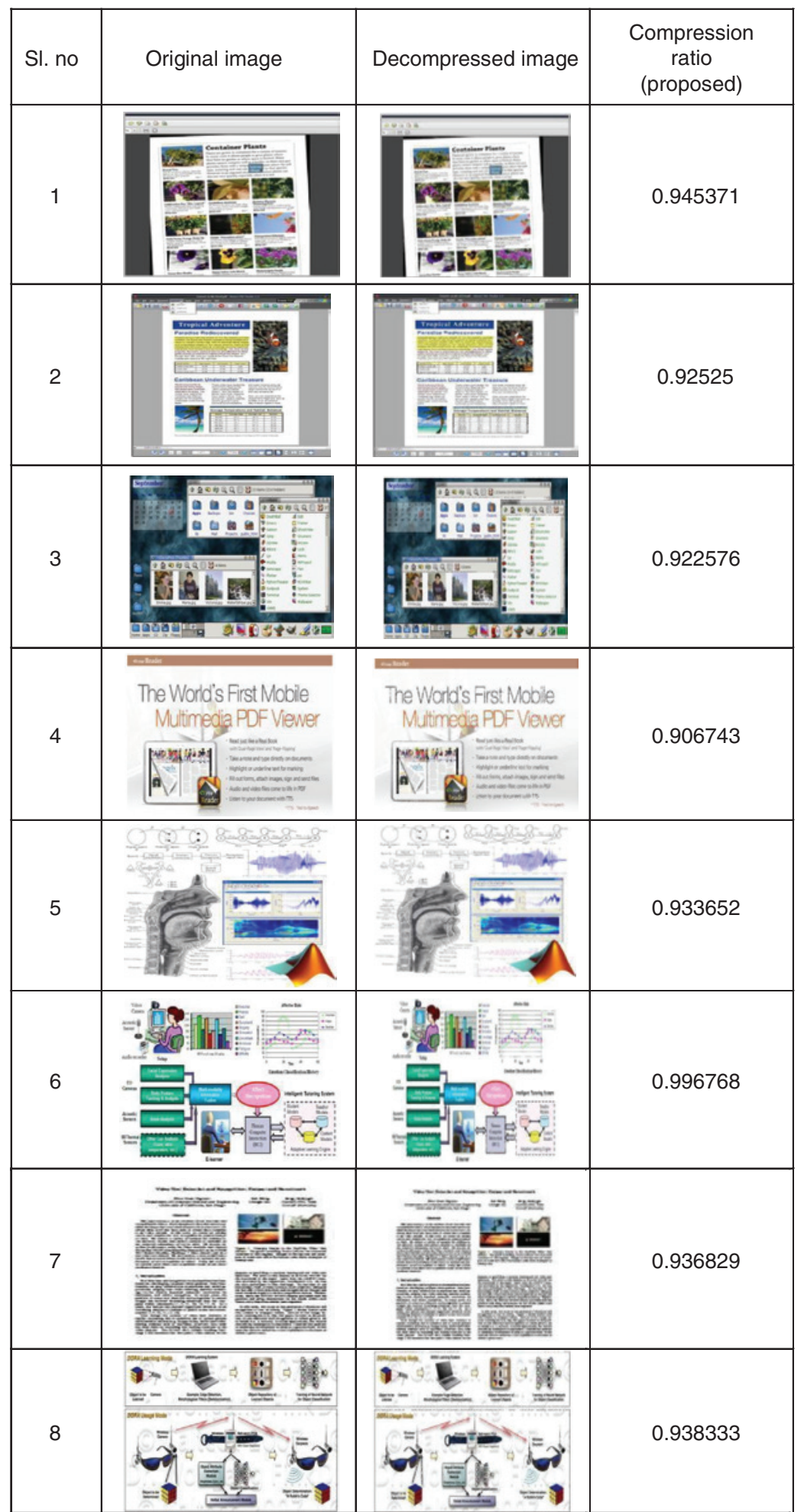

developing which gives better CR. In this compression process, DWT with Harr coefficients, optimization process and RLC coding is used. As the CR is increased, the value of PSNR will decrease. This will degrade the quality of the image, and the DWT will provide better compression results at low CR. From the experimental results, it is evident that the proposed compression technique gives better performance compared to other 
Table 2: File Size Comparison for Proposed Method.

\begin{tabular}{lrrrr}
\hline Images & Original size (bytes) & Compressed size (bytes) & MSE & PSNR \\
\hline 1 & 15,285 & 14,450 & 5.588976 & 40.65749 \\
2 & 18,796 & 17,391 & 5.594714 & 40.65303 \\
3 & 12,903 & 11,904 & 4.403576 & 41.69283 \\
4 & 13,940 & 12,640 & 4.866342 & 41.25893 \\
5 & 17,363 & 16,211 & 5.438889 & 40.77571 \\
6 & 14,234 & 14,188 & 6.71798 & 39.85842 \\
7 & 17,318 & 16,224 & 5.340546 & 40.85496 \\
8 & 15,162 & 14,227 & 5.819019 & 40.48232 \\
\hline
\end{tabular}

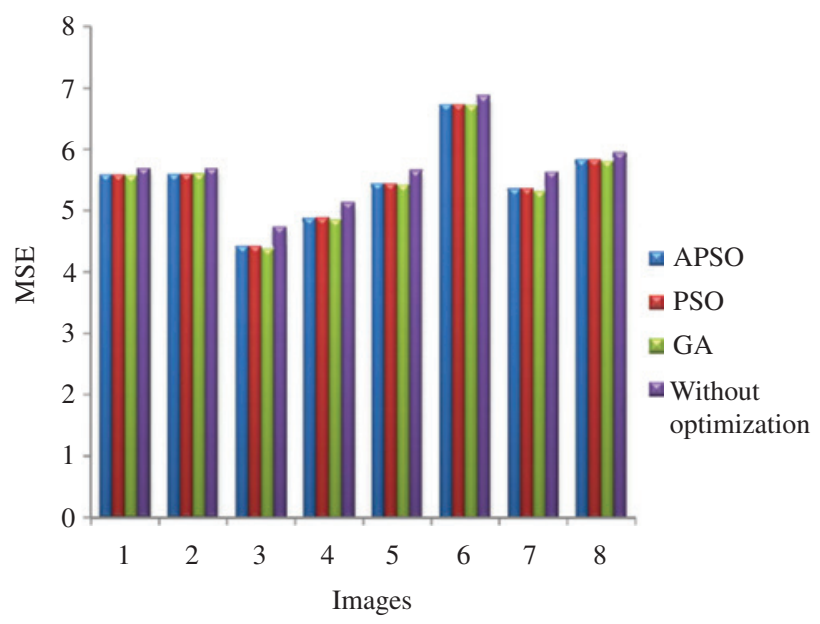

Figure 7: MSE Comparison.

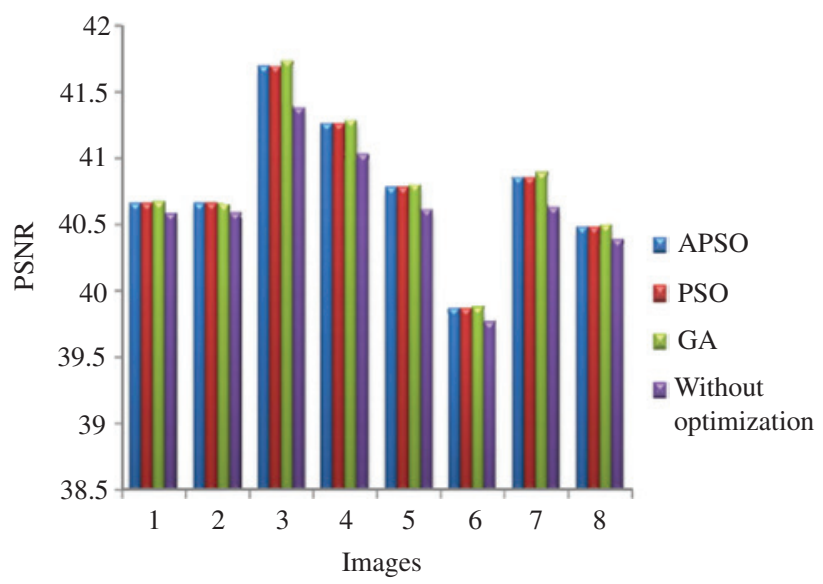

Figure 8: PSNR Comparison.

traditional techniques. The maximum PSNR of the image is 40.771 and CR is 0.93 in the optimized wavelet image compression process. Wavelets are better suited to time-limited data, and wavelet-based compression technique maintains better image quality by reducing errors. A few implications in view of this paper are included: The adequacy and vigor of this approach has been justified as utilizing an arrangement of real images. The compression techniques utilized here are basic, computationally less mind boggling and furthermore more proficient in the part of time execution. Wavelet transform can be connected to any image as many 


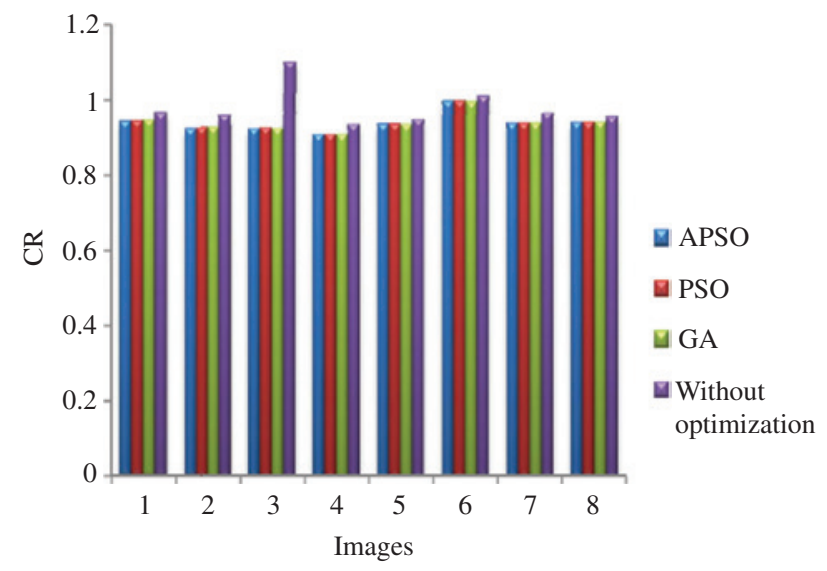

Figure 9: Comparison Ratio Comparison.

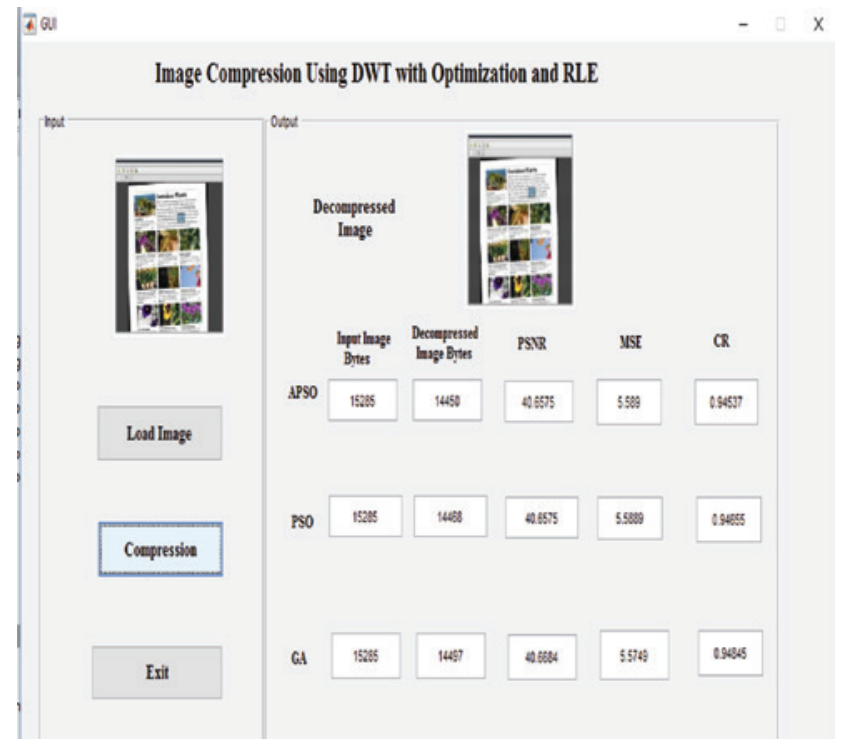

Figure 10: Graphical User Interface (GUI) in MATLAB.

circumstances as could be allowed, and the subsequently high compression proportion is accomplished. Wavelet-based coding enhances picture quality, and it is vigorous against transmission blunder. It additionally gives an effective disintegration of the image before compression and permits low piece rate transmission. It is observed that the proposed algorithm has preferable execution over JPEG compression in low and high bit rates. Additionally, the proposed compression plot has normally unrivaled performance in the image. In compound image compressing strategies, compression with the assistance of a solitary algorithm that all the while meets the necessities for content, image and illustrations has been ambiguous. Occasionally, the individual algorithm does not require ideal compression process, and the procedure might be complex. Thus, in future work we will utilize new hybrid inventive algorithms that can skillfully decrease the file size without corrupting the quality. The future direction of this research is to implement a compression technique using other approaches. 


\section{Bibliography}

[1] C. Agulhari, I. Bonatti and P. Peres, An adaptive run length encoding method for the compression of electrocardiograms, J. Med. Eng. Phys. 35 (2013), 145-153.

[2] R. Baumeister, U. Held, M. Thali, P. Flach, and S. Ross, Forensic imaging findings by post-mortem computed tomography after manual versus mechanical chest compression, J. Forensic Radiol. Imaging. 3 (2015), 167-173.

[3] M. Boix and B. Canto, Wavelet transform application to the compression of images, J. Math. Comput. Model. 52 (2010), 1265-1270.

[4] T. Bruylants, A. Munteanu, and P. Schelkens, Wavelet based volumetric medical image compression, J. Sign. Proc. Image Commun. 31 (2015), 112-133.

[5] H.-L. Chana, Y.-C. Siao, S.-W. Chen and S.-F. Yu, Wavelet-based ECG compression by bit-field preserving and running length encoding, J. Comput. Methods Programs Biomed. 90 (2008), 1-8.

[6] M. H. Chowdhury and A. Khatun, Image compression using discrete wavelet transform, J. Comput. Sci. 9 (2012), $327-330$.

[7] H. Z. Eldin, M. Elhosseini and H. Ali, Image compression algorithms in wireless multimedia sensor networks: a survey, J. Ain Shams Eng. 6 (2014), 1-10.

[8] R. Jafari, D. Ziou and M. M. Rashidi, Increasing image compression rate using steganography, J. Expert Syst. Appl. 40 (2013), 6918-6927.

[9] X. Kai, Y. Jie, Z. Y. Min and L. X. Liang, HVS-based medical image compression, J. Radiol. 55 (2005), 139-145.

[10] S. Kaur, G. Kaur and D. Singh, A review: various wavelet based image compression techniques, J. Sci. Res. 2 (2013), 1-4.

[11] S. Khuri and H.-C. Hsu, Interactive packages for learning image compression algorithms, J. ACM SIGCSE Bull. 32 (2000), 73-76.

[12] J. Li, An improved wavelet image lossless compression algorithm, J. Optik. 124 (2013), 1041-1044.

[13] H.-S. Li, Q. Zhu, R. Guizhou, M.-C. Li, L. Song and H. Ian, Multidimensional color image storage, retrieval, and compression based on quantum amplitudes and phases, J. Inform. Sci. 273 (2014), 212-232.

[14] J. M. Pascual, H. M. Mora, A. F. Guillo and J. A. Lopez, Adjustable compression method for still JPEG images, J. Image Commun. 32 (2015), 1-18.

[15] P. Peter, C. Schmaltz, N. Mach, M. Mainberger and J. Weickert, Beyond pure quality: progressive modes, region of interest coding, and real time video decoding for PDE-based image compression, J. Vis Commun Image Retrieval. 31 (2015), 253-265.

[16] S. Saadi, M. Touiza, F. Kharfi and A. Guessoum, Dyadic wavelet for image coding implementation on a Xilinx MicroBlaze processor: application to neutron radiography, J. Appl. Radiat. Isotopes 82 (2013), 200-210.

[17] V. Setia and V. Kumar, Coding of DWT coefficients using run-length coding and Huffman coding for the purpose of color image compression, J. Comput. Commun. Eng. 6 (2012), 201-204.

[18] S. Sidhik, Comparitive study of Birge-Massart strategy and unimodal thresholding for image compression using wavelet transform, Int. J. Light Electron Optics. 126 (2015), 1-6.

[19] H. Singh and S. Sharma, Hybrid image compression using DWT, DCT \& Huffman encoding techniques, J. Emerg. Technol. Adv. Eng. 2 (2012), 300-306.

[20] S. K. Singh and S. Kumar, Novel adaptive color space transform and application to image compression, J. Sign. Proc. Image Commun. 26 (2011), 662-672.

[21] B. Wang, X. Zheng, S. Zhou, C. Zhou, X. Wei, Q. Zhang and C. Che, Encrypting the compressed image by chaotic map and arithmetic coding, J. Optik. 125 (2014), 6117-6122.

[22] M.-S. Wu, Genetic algorithm based on discrete wavelet transformation for fractal image compression, J. Vis. Commun. Image Retrieval. 25 (2014), 1835-1841.

[23] C.-H. Yuen and K.-W. Wong, A chaos-based joint image compression and encryption scheme using DCT and SHA-1, J. Appl. Soft Comput. 11 (2011), 5092-5098.

[24] N. Zhou, A. Zhang, F. Zheng and L. Gong, Novel image compression-encryption hybrid algorithm based on key-controlled measurement matrix in compressive sensing, J. Optics Laser Technol. 62 (2014), 152-160.

[25] Z. Zuo, X. Lan, L. Deng, S. Yao and X. Wang, An improved medical image compression technique with lossless region of interest, Int. J. Light Electron Optics 26 (2015), 1-22. 